\title{
Aplicación de las herramientas de traducción asistida por ordenador (TAO) a la investigación en traducción
}

\author{
Montserrat Bermúdez Bausela \\ Universidad Alfonso X el Sabio \\ mbermbau@uax.es
}

Recibido: 15 de octubre de 2013

Aceptado: 18 de febrero de 2014

\section{RESUMEN}

En este artículo vamos a ofrecer una serie de pautas sobre cómo se puede aplicar una herramienta de traducción asistida por ordenador (TAO) a una labor de investigación en el ámbito de los Estudios de Traducción. Para ello, hemos delimitado la aplicación del estudio a una muestra textual formada por textos originales y sus respectivas traducciones de una naturaleza especializada. Todo ello desde una aproximación descriptiva, comunicativa y pragmática. La herramienta TAO escogida para ejemplificar esta utilidad es Trados WinAlign, una herramienta de alineación de textos integrada en el paquete de herramientas de ayuda al traductor Trados. Veremos cómo se prepara la muestra, cómo se segmenta y se deja lista para, posteriormente, analizar sobre ella el trasvase interlingüístico de ciertas unidades que se escojan para el estudio.

Palabras clave: herramientas de traducción asistida por ordenador (TAO), muestra textual, estudios descriptivos, normas de traducción.

\section{Implementation of Computer Assisted Translation Tools (CAT) to research in the field of Translation Studies.}

\begin{abstract}
In this article we are going to offer a number of guidelines on the implementation of a Computer Assisted Tool (CAT) in research within the field of Translation Studies. With this aim in mind, we have delimited the study to a textual sample composed of specialized source texts and their translations. The approach is a descriptive, communicative and pragmatic one. The chosen CAT Tool to exemplify its usefulness is Trados WinAlign, a text alignment tool, integrated in the set of Trados tools. We will show how to prepare the sample, how to segment it and once it is ready, the purpose would be to carry out an analysis of the interlinguistic process of some specific units.
\end{abstract}


Keywords: Computer Assisted Tools (CAT Tools), textual sample, descriptive studies, translation norms.

Sumario: 1. Introducción. 2. Herramientas de Traducción Asistida por Ordenador (TAO). 3. Marco de investigación: los Estudios de Traducción de corte descriptivo y las muestras textuales. 4. Análisis y ejemplificación. 4.1 Objetivos y metodología del estudio. 4.2 Preparación y procesamiento informático de la muestra textual. 4.3 Incorporación de los resultados a una base de datos. 5. Conclusiones.

\section{Introducción}

Las herramientas de traducción asistida por ordenador (TAO) están demostrando ser de gran utilidad en el campo de investigación de los Estudios de Traducción, especialmente en los estudios de corte descriptivo y aplicados a muestras textuales (compuestas por originales y sus respectivas traducciones) que el investigador reúne para llevar sobre ellas un estudio concreto. El fin de este tipo de investigación es, normalmente, el de detectar una serie de regularidades, a partir siempre de la observación del proceso traductor, para poder extraer unas normas de traducción, o, al menos, ser capaces de ofrecer una serie de pautas sobre cómo traducir un tipo específico de textos. Además, este tipo de estudios suele llevarse a cabo sobre textos de especialidad más que sobre textos de naturaleza general.

Para demostrar su utilidad, vamos a señalar cómo podría llevarse a cabo este tipo de estudio en una muestra textual compuesta por originales en inglés y sus traducciones al español, la cual se ha de preparar, como paso previo a la investigación, llevándose a cabo sobre ella un proceso de segmentación y alineación para, posteriormente, identificar y estudiar el comportamiento en traducción de las unidades de estudio que se hayan fijado como objeto de análisis. Para los fines de este artículo, nos vamos a limitar a explicar la preparación de la muestra textual.

Para ello, hemos organizado nuestro artículo en varias secciones. En primer lugar, ofrecemos un breve recorrido por las principales herramientas de traducción asistida por ordenador. A continuación, delimitaremos el ámbito de investigación. En este último punto abordaremos aspectos como los estudios de corte descriptivo aplicados a las muestras textuales, para, finalmente, concretar cómo se puede preparar la muestra textual con estas herramientas.

\section{Herramientas de Traducción Asistida por Ordenador (TAO)}

Son numerosos los tipos de herramientas comúnmente denominadas herramientas de Traducción Asistida por Ordenador (TAO) ${ }^{1}$, además de ser también numero-

${ }^{1} \mathrm{O}$ también herramientas TEnT (Translation Environment Tools) por su referencia al entorno de traducción. Este término fue acuñado por Zetzsche (2003). En inglés estas herramientas se suelen denominar CAT Tools (Computer Assisted Translation Tools). 
sas sus propuestas de clasificación atendiendo a distintos criterios tales como la distinción entre traducción automática (que no se consideraría en ese sentido herramienta TAO) frente a las memorias de traducción (cf. Hutchins \& Somers 1992); atendiendo a la fase del proceso de traducción en el que estas herramientas se emplean, bien sea de forma directa o indirecta, sin importar si se utilizan antes, después o durante el proceso (como por ejemplo, la preparación documental, la conversión de formatos o la maquetación) (cf. Melby 1998); o según la función que estas herramientas desempeñan (cf. Alcina 2008).

Así, en nuestro caso, vamos a considerar herramientas TAO aquellas que atañen directamente al trabajo del traductor, entre ellas:

1. Memorias de traducción (TM): como por ejemplo SDL Trados (en sus varias versiones, destacando la actual SDL Trados Studio 2014, pero sin dejar de mencionar la anterior SDL Trados 2007), Déjà Vu X2, Star Transit, así como memorias de traducción que han saltado últimamente al mercado y se han hecho con un hueco inmediato debido a su eficiencia y versatilidad, como, por ejemplo, MemoQ, Wordfast, además de la gratuita OmegaT. Una memoria de traducción es un tipo de software que sugiere al traductor traducciones previamente almacenadas en la memoria que presentan un porcentaje de correspondencia determinado respecto al nuevo segmento textual que se ha de traducir.

2. Gestores terminológicos: prácticamente la mayoría de memorias de traducción tienen incorporado su propio gestor terminológico, como es el caso de DéjàVuX2 (Terminology Maintenance) y SDL Trados (MultiTerm).

3. Herramientas de alienación de textos: cuya finalidad es la de alinear originales con sus respectivas traducciones con el objetivo de alimentar una memoria de traducción. Un ejemplo de esta herramienta es SDL Trados WinAlign.

4. Herramientas de localización de software: son herramientas utilizadas en la traducción y adaptación de productos informáticos. Ejemplos: SDL Passolo y Alchemy Catalyst.

5. Herramientas de localización de páginas web: como por ejemplo CatsCraddle y WebBudget. Se trata de editores de páginas web que nos permiten traducir páginas web sin peligro de borrar alguna etiqueta del código HTML.

6. Herramientas de subtitulación: que nos permiten incluir los subtítulos en la traducción audiovisual. Un ejemplo es Subtitle Workshop.

7. Herramientas de ayuda lexicográfica o explotación de corpus: este tipo de herramientas, entre las que destacamos WordSmith Tools, nos permite realizar concordancias, búsquedas de colocaciones, ver agrupaciones de palabras, extraer listados de palabras, etc., para el estudio del léxico y la fraseología, entre muchas otras utilidades.

Sin olvidarnos de otros recursos y herramientas electrónicas que, sin considerarse tal vez herramientas TAO propiamente dichas, también están muy vinculadas al mundo de la traducción, tales como las herramientas de gestión de proyectos, la cuales nos permiten gestionar proyectos de traducción, como por ejemplo MS Project; 
las herramientas de maquetación como FrameMaker, QuarkXPress y Adobe InDesign, cuyo objetivo es que el aspecto final responda a las necesidades del encargo de traducción, así como todos aquellos recursos de ayuda en línea tan útiles para el traductor. Más controvertido es el uso de las máquinas de traducción (MT), entre las que destacamos Systran. Las máquinas de traducción, al contrario que las memorias de traducción, ofrecen una traducción automática del texto introducido por el usuario. Este texto posteriormente ha de ser editado. En principio, es más recomendable para un traductor una memoria de traducción que una máquina de traducción, dado que en la memoria de traducción, las unidades almacenadas proceden de traducciones previas, no de una máquina. Por lo que respecta a las máquinas de traducción, estas suelen ser de mayor utilidad en empresas en las que se utiliza un lenguaje controlado. Un lenguaje controlado siempre está limitado en cuanto al léxico utilizado y sus estructuras gramaticales ${ }^{2}$.

En nuestro caso, la herramienta que vamos a utilizar para el caso práctico es la herramienta de alineación Trados WinAlign. Pero, independientemente de la que hayamos escogido, lo relevante es ver cómo las herramientas TAO se pueden utilizar en beneficio de la actividad investigadora en traducción, por lo que, a continuación, vamos a describir un posible marco en el que creemos que podría tener cabida el uso de estas herramientas.

\section{Marco de investigación: los Estudios de Traducción de corte descriptivo y las muestras textuales}

Partimos de la consideración de que las normas y convenciones textuales tienen un impacto directo en el proceso de traducción, muy especialmente en los lenguajes de especialidad. En este sentido, estudiosos procedentes del campo de la traducción y de los estudios contrastivos han hecho hincapié, por un lado, en la relevancia y repercusión de las diferencias retóricas en el trasvase interlingüístico y, por otro, en su influjo en la redacción académica en una segunda lengua. Asimismo, creemos que el estudio del tipo de relación de equivalencia entre originales y sus traducciones en un contexto real de traducción puede aportar datos valiosos para la labor del traductor que, esperamos, se manifiesten en una serie de rasgos contrastivos distintivos.

En este artículo planteamos un marco de investigación de corte descriptivo en el que el objetivo es describir y explicar cualquier tendencia y regularidad observada en el proceso traductológico en torno al tratamiento de ciertas unidades sobre las que el investigador desee llevar a cabo su labor. Estas unidades podrían pertenecer tanto a la macroestructura como a la microestructura textual. Además, pensamos que este tipo de investigación aportaría unos resultados más notorios en un contexto de tra-

\footnotetext{
2 Mencionamos algunos de estos proyectos, como por ejemplo es el Caterpillar Fundamental English (cuya versión más reciente es el Caterpillar Technical English), el AECMA Simplified English, utilizado por la industria aeroespacial, y, por último, el Perkins (Perkins Approved Clear English, PACE) (cf. Amores Carredano, 2000).
} 
ducción especializada, que situaría el comportamiento traductor hacia una adecuación a las normas retóricas de la lengua y de la cultura origen o, por el contrario, hacia una adaptación a las convenciones textuales de la lengua y cultura meta ${ }^{3}$. Recordemos, brevemente, en este punto, el concepto de "norma" expuesta por Toury (1978), central en los Estudios de Traducción, y procedente del ámbito de la sociología:

[...] the translation of general values or ideas shared by a certain community -as to what is right and wrong, adequate or inadequate- into specific performance instructions appropriate for and applicable to specific situations, providing they are not (yet) formulated as laws (Toury 1978: 83).

Las normas, en este sentido, son pautas, convenciones aceptadas por una comunidad determinada que derivan en la observación de regularidades en el comportamiento del grupo social, por lo que "it is only natural that such regularities should serve as the main evidence in the study of norms" (Toury 1978: 84). Aplicadas a la traducción, las normas regulan y determinan la actividad traductora (el comportamiento traductor) "at every stage in the translating process and at every level in its product, the translation itself” (Toury 1978: 86) en lo referente a la relación entre el original y la traducción, a la selección de textos que se han de traducir, a la estrategia global a seguir, etc.

Otros autores exponentes de este enfoque descriptiva son Lambert \& Van Gorp (1985) o Lefevere (1992). Estos teóricos otorgan relevancia al estudio de las normas que rigen el proceso de traducción, puesto que es fundamental determinar las normas predominantes en el sistema meta para entender la naturaleza del trasvase que se ha realizado. En el caso español, Rabadán Álvarez (1991) entiende la equivalencia en el marco general de la teoría del polisistema (cf. Even-Zohar 1979). Su concepto de equivalencia "translémica" es un concepto de carácter funcional y relacional, referido a la relación global, única e irrepetible que se establece para cada binomio textual texto origen (TO)-texto meta (TM) y en la que las normas traductológicas juegan un papel fundamental. En este contexto, lo que permite definir un TM como traducción de un TO es su aceptabilidad por parte del receptor del polisistema meta.

En efecto, es importante que el investigador trabaje con traducciones equivalentes a nivel textual y comunicativo, que ya hayan sido aceptadas como traducciones válidas en el contexto de recepción, por lo que entre los posibles objetivos de la investigación estaría explicar la influencia de los factores de producción textual en el tipo de equivalencia que situaría las traducciones de la muestra en el continuo de la adecuación o de la aceptabilidad.

\footnotetext{
3 Los términos de adecuación y aceptabilidad se han adoptado de la teoría del polisistema (cf. EvenZohar 1979) por Santoyo Mediavilla \& Rabadán Álvarez (1991). Rabadán Álvarez habla del “espacio intersubjetivo" (1991).
} 
Como puede observarse, para los propósitos de este artículo, nos identificamos más con las aproximaciones descriptivas ${ }^{4}$ de tipo comunicativo, en las que se basa nuestra concepción de la traducción. Creemos que la Traducción entendida como ciencia empírica ha de contemplar entre sus objetivos la identificación de patrones de respuesta a situaciones de traducción específicas por parte de los traductores (cf. Neubert \& Shreve 1992: 148; Toury 1995:65-66) así como la descripción y explicación de la conducta traductora a través de las propias traducciones, que constituyen en sí mismas la mejor realidad observable:

Translation studies in its empirical form are primarily descriptive and should be based on the observation of translation practice. As an empirical science, it has two objectives. It seeks to identify regularities in the way that translators respond to specific translation situations, and it seeks to identify regularities in the results of that response (Neubert \& Shreve 1992: 8).

Creemos que para llevar a cabo un estudio de este tipo, descriptivo y pragmático, puede resultar especialmente útil la delimitación de una muestra textual sobre la que llevar a cabo la investigación. Hablamos en todo momento de "muestra textual" y no de "corpus lingüístico" dado que se trata de una compilación de textos mucho más reducida y que aspira únicamente a dar cuenta de una pequeña realidad traductora.

Para resumir lo expuesto anteriormente, el investigador delimitaría su trabajo al estudio del trasvase lingüístico de ciertas unidades de la microestructura o macroestructura en una muestra textual formada por originales y sus traducciones pertenecientes a un ámbito de especialidad con el fin de observar regularidades en el proceso de traducción. A continuación, veamos cómo se podría plantear esta labor y cómo nos podríamos ayudar de las herramientas TAO para nuestros fines.

\section{Análisis y ejemplificación}

\subsection{Objetivos y metodología del estudio}

Entre los posibles objetivos de un estudio descriptivo de estas características podríamos establecer los siguientes:

${ }^{4}$ Las premisas fundamentales de las que parte el descriptivismo como aproximación a los estudios de traducción es la consideración de aspectos textuales, pragmáticos, funcionales, culturales y contextuales en el proceso traductivo, puesto que son aproximaciones de tipo comunicativo para las que no existen condiciones "ideales" en traducción sino condiciones "reales". Además, como su nombre indica, estos estudios se centran en describir la naturaleza de la traducción, no en prescribir cómo se ha de traducir: se interesan por las normas que intervienen en la producción y recepción de las traducciones (Hermans 1985: 10-11) y propugnan modelos target-oriented centrados en el receptor del TM y la función que ha de cumplir el TM en su nuevo contexto. 
1. Análisis de un conjunto de tendencias traductológicas extraídas de la muestra textual.

2. Estudio del papel discursivo de ciertos elementos textuales.

3. Estudio de la repercusión de las diferentes preferencias discursivas en la práctica traductora.

4. Aportación de valiosos datos sobre estilística contrastiva.

5. Contribución a la especificación de la norma inicial de traducción que prevalece en el trasvase interlingüístico de los textos especializados estudiados.

6. Aportación de datos relevantes sobre las preferencias discursivas en la muestra textual para las dos lenguas implicadas en la investigación.

Por lo que se refiere a la metodología de la investigación, sobre una muestra textual se puede llevar a cabo un estudio descriptivo basado en el análisis de géneros profesionales y académicos en un contexto especializado de traducción. Entre algunas de las recomendaciones sobre cómo llevar a la práctica este tipo de estudio podríamos mencionar las siguientes:

1. Compilación de una muestra textual que responda a unos criterios homogéneos: por ejemplo, textos que se circunscriban a un ámbito de especialidad (normalmente jurídico, económico y comercial).

2. Selección de determinados aspectos de la macroestructura o de la microestructura sobre los que se desea llevar a cabo el estudio para observar cómo se produce su trasvase lingüístico en el par de lenguas escogidas.

3. Estudio de todos aquellos factores situacionales y pragmáticos que rodean al proceso de producción y traducción de los textos.

4. Identificación del original y sus traducciones.

5. Consideración de la posibilidad de trabajar con herramientas de traducción asistida por ordenador para la gestión de la muestra textual, por ejemplo, el empleo de Trados WinAlign, para la alineación de los textos paralelos.

6. La creación de una base de datos donde se vayan incluyendo todos los ejemplos encontrados de aquellas unidades textuales objeto de estudio para su posterior análisis.

7. Estudio y análisis de los datos.

8. Interpretación de los resultados del análisis y valoración de los resultados obtenidos.

9. Posible extracción de una serie de regularidades observadas en el proceso de traducción.

10. Establecimiento de una serie de tendencias traductológicas, rasgos o patrones repetitivos que se puedan incorporar a una guía como futura ayuda al traductor de este tipo concreto de textos especializados.

\subsection{Preparación y procesamiento informático de la muestra textual}

Por lo que respecta a la preparación de la muestra textual, utilizaremos una herramienta específica de alineación de textos denominada WinAlign, integrada en 
la herramienta de traducción asistida por ordenador Trados. Esta herramienta nos permite alinear un TM con su correspondiente TO, para lo que previamente divide cada texto en segmentos y después los alinea. La alineación de los textos se va a convertir, en sí misma, en la muestra textual sobre la que llevar a cabo nuestro análisis. Una vez tenemos la muestra textual accesible y preparada para ser manipulada y poder trabajar con ella, el investigador procedería a la identificación de las unidades objeto de estudio. Para la recogida de información, en este artículo recomendamos la creación de una base de datos que, a partir de una serie de campos que se definan, sea capaz de categorizar, de la forma más precisa posible, las unidades de estudio y, así, poder posteriormente analizar e interpretar los datos.

A continuación procedemos a explicar los pasos que se han seguido en la preparación de la muestra textual para su segmentación y alineación. Como decíamos previamente, Trados WinAlign nos permite alinear un TM con su correspondiente TO, para lo que previamente divide cada texto en segmentos y después los alinea. Para realizar la segmentación, el programa se guía a partir de marcas como es el punto $<$., , los dos puntos $\langle:>$, la marca de tabulador $\langle$ Tab $>$, o la señal de fin de párrafo $\langle\mathbf{q}>$, entre otros criterios que el usuario selecciona previamente (cf. Figura 1). WinAlign también reconoce como segmentos independientes los títulos y los elementos de una numeración o de una viñeta.

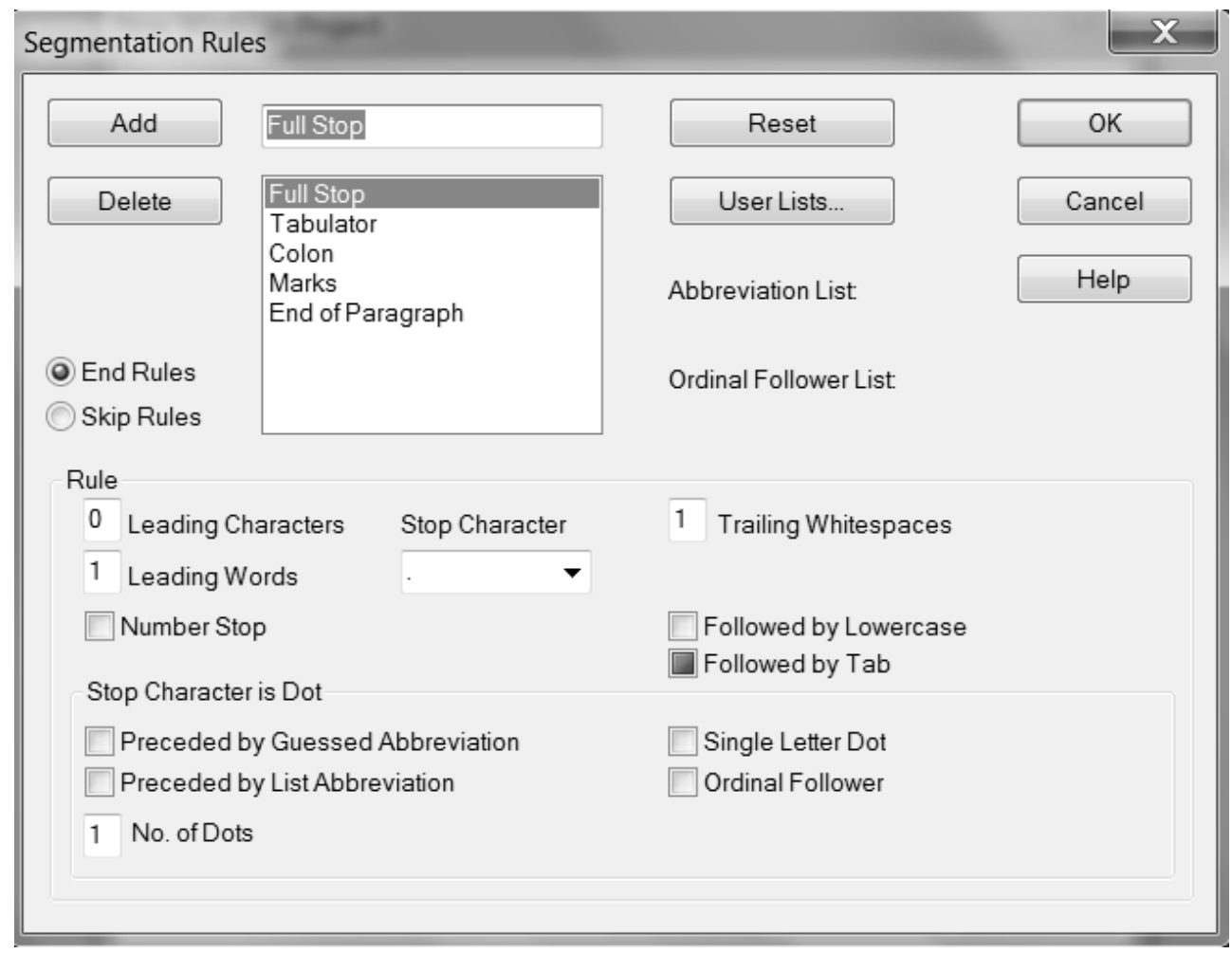

Figura 1: Reglas de segmentación 
En primer lugar, es preciso guardar los documentos en formato enriquecido de texto (RTF), uno de los formatos requeridos para trabajar con esta herramienta ${ }^{5}$. Cuando se transforma un archivo en formato PDF o HTML a formato RTF, se producen algunas modificaciones de formato (cambio de tamaño y tipo de letra, especialmente), diferencias de alineamiento y de saltos de línea, algo que dificulta enormemente el correcto alineamiento de los segmentos, por lo que es tarea ardua, aunque imprescindible, revisar los textos e igualarlos en cuanto al formato, para que su posterior segmentación resulte más sencilla. No obstante, es importante para los objetivos de la investigación no variar ni adulterar la estructura de los textos. Aconsejamos crear un nuevo proyecto de alineación para cada uno de los textos (con su correspondiente traducción) que componen la muestra textual (cf. Figura 2):

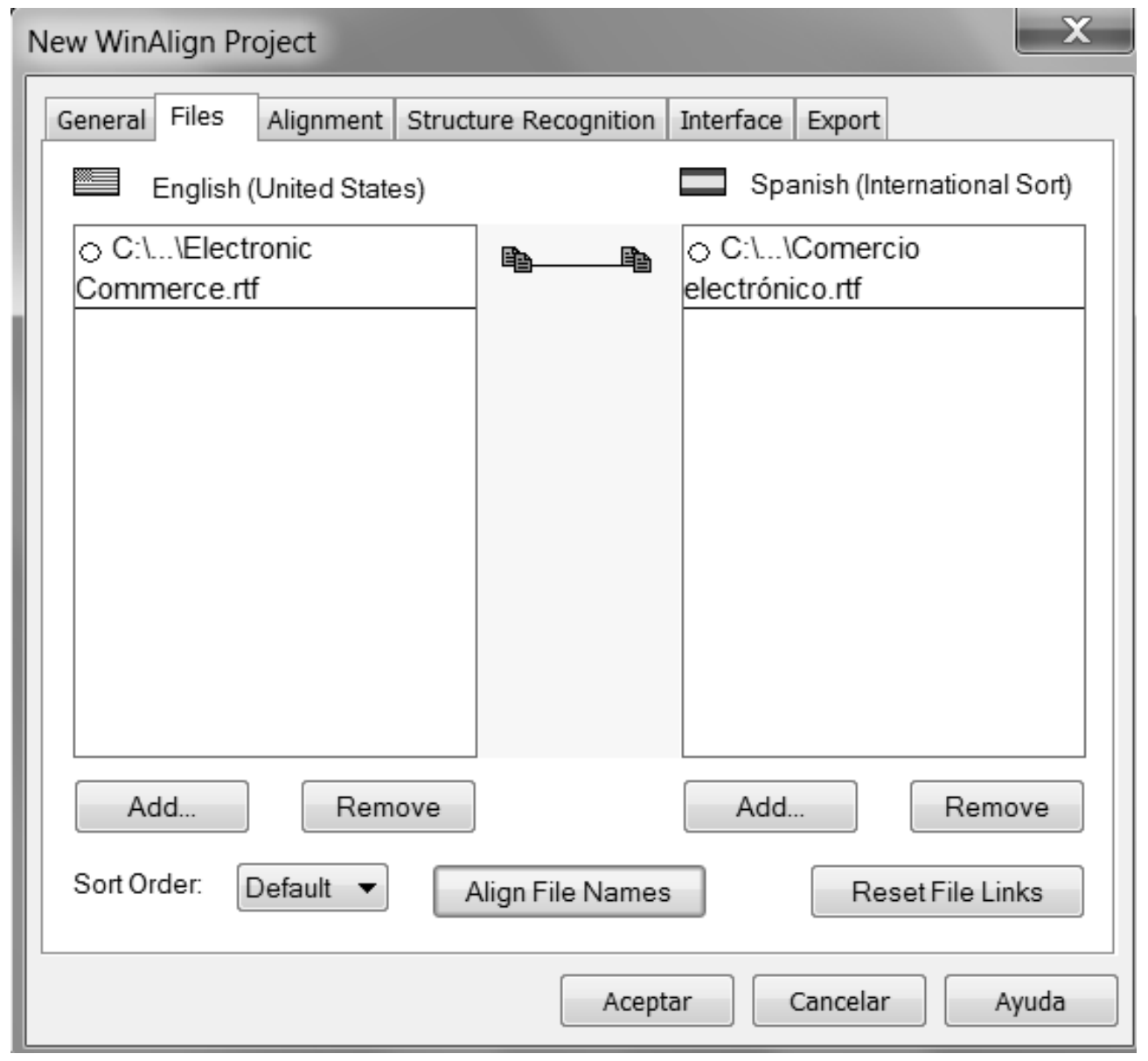

Figura 2: Nuevo proyecto de alineación

125 No es el único, pero sí el más recomendable. 
Es preciso apuntar que es posible trabajar con WinAlign siempre que la macroestructura de los originales y traducciones sea muy similar, a ser posible idéntica, de lo contrario, la segmentación sería una tarea prácticamente imposible.

Una vez que se han especificado los parámetros de alineación y reconocimiento de la estructura, los textos están preparados para comenzar el proceso de alineación. Una vez se han alineado es necesario revisar cada unidad de traducción ${ }^{6}$ (cf. Figura 3, Figura 4). Para Trados, el objetivo de este proceso es alimentar la memoria de traducción mediante la exportación de las unidades de traducción resultantes a un archivo en formato "solo texto" (TXT) ${ }^{7}$. Sin embargo, a nosotros nos interesa la exportación de estos segmentos por otros motivos, ya que la alineación de los textos se va a convertir, en sí misma, en la muestra textual sobre la que llevar a cabo nuestro análisis.

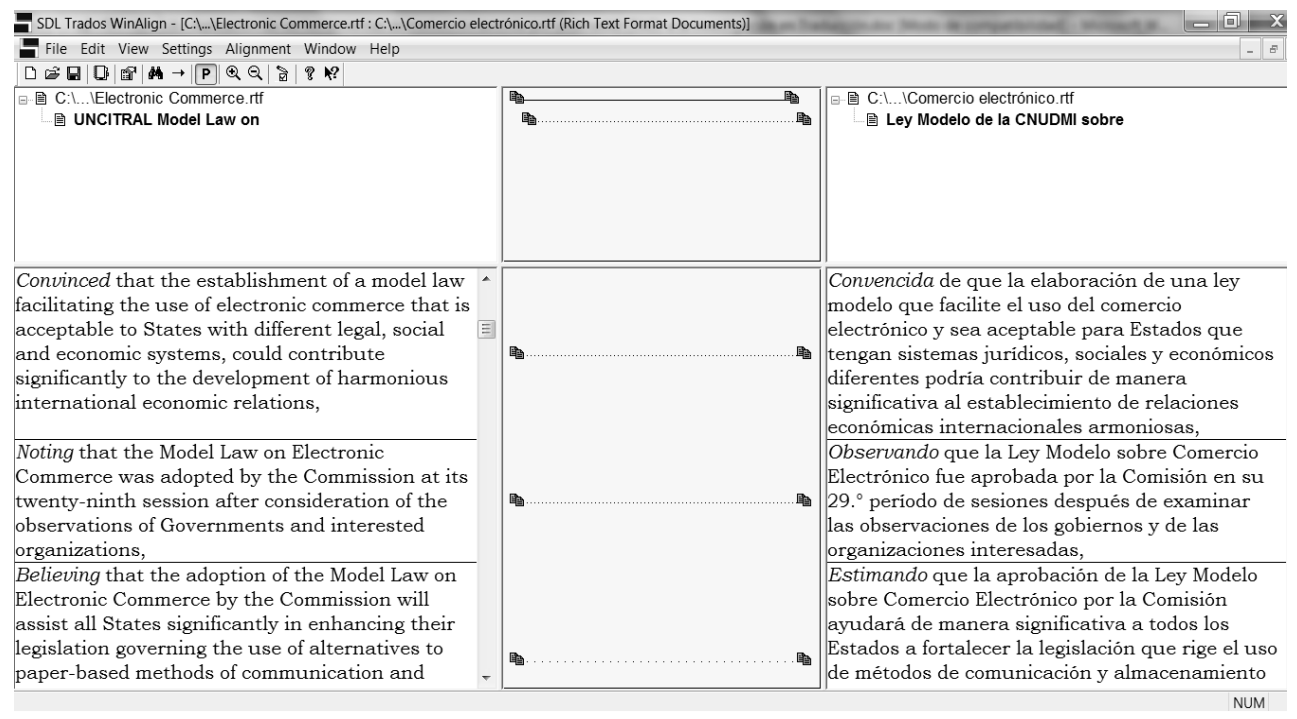

Figura 3: Revisión de la alineación I

${ }^{6}$ Por unidad de traducción se entiende en WinAlign el par de segmentos formados por el segmento origen y su correspondiente traducción.

7 Creemos que debemos hacer una puntualización. Como hemos dicho, una vez que hemos realizado la alineación de segmentos originales con sus traducciones utilizando WinAlign, la herramienta exporta las unidades de traducción resultantes de la alineación a un archivo en formato TXT. Este archivo en formato TXT es posteriormente importado a la memoria de traducción. No debemos confundir este procedimiento ni este formato con este otro: el formato TMX (Translation Memory eXchange), un estándar de XML utilizado para el intercambio de documentos entre distintas memorias de traducción que trabajan con formatos diferentes. 


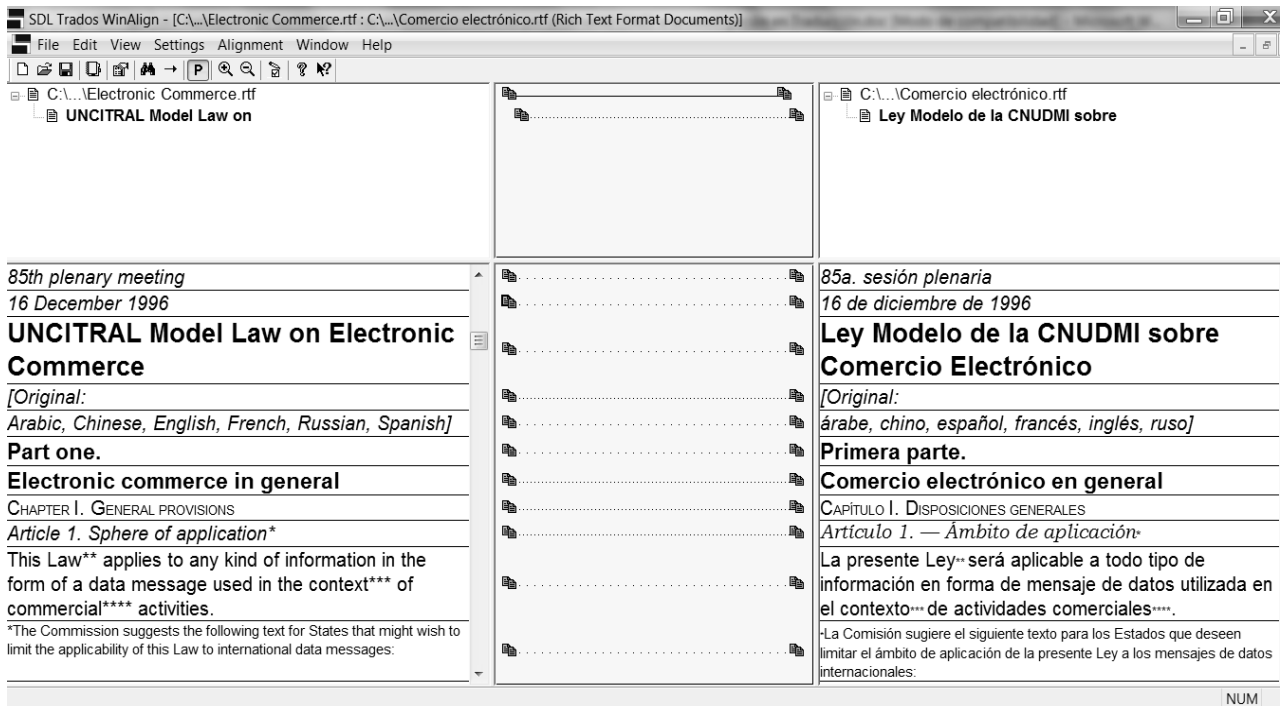

Figura 4: Revisión de la alineación II

La utilidad del proyecto de alineación creado y guardado con WinAlign (PJT) una vez que se ha exportado el archivo que contiene todos los pares de segmentos alineados es nula, ya que en el momento en que se cambia la ubicación de los archivos, algo inevitable a lo largo del proceso de investigación, ya no hay posibilidad de volver a recargar la alineación en el programa de WinAlign. Sin embargo, esto no es un obstáculo, ya que lo que nos interesa es la exportación del archivo TXT (cf. Figura 5) en sí mismo, que, como hemos dicho, es el resultado de la alineación de los segmentos, corregida y verificada.

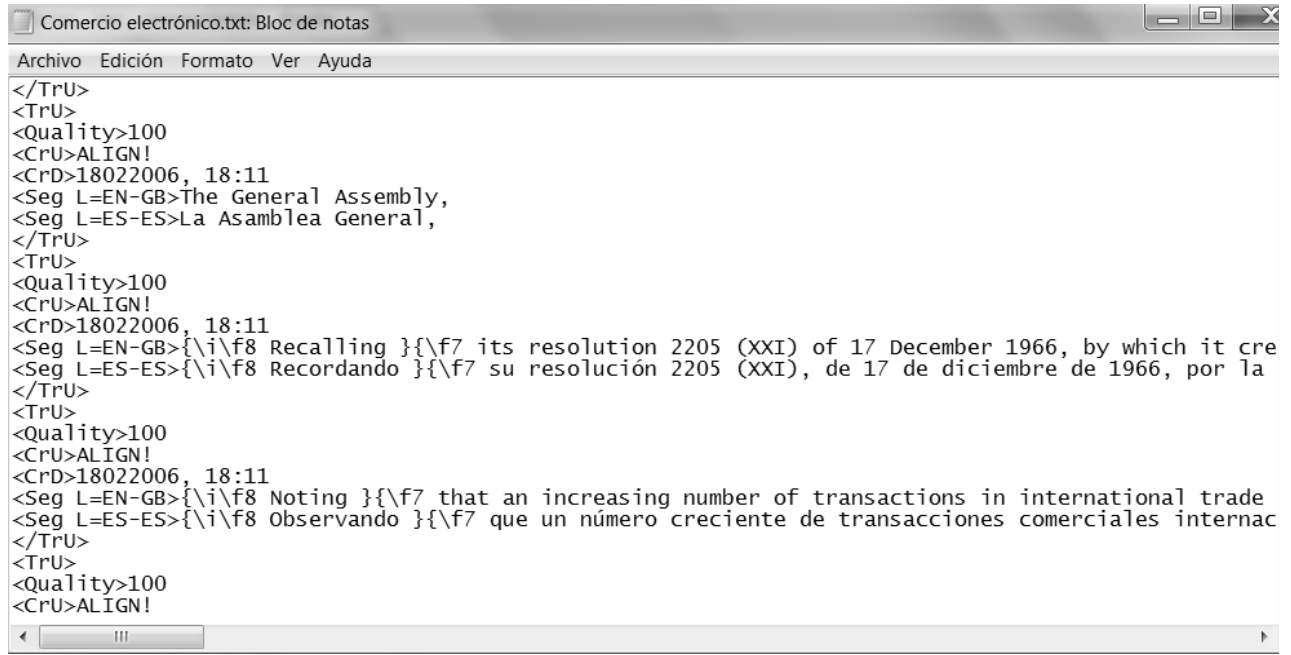

Figura 5: Archivo en formato TXT

Estudios de Traducción

2014, vol. 4, 129-143 
Cuando obtenemos el archivo en formato TXT lo convertimos a formato DOC (formato del procesador de datos Word) para mayor facilidad en el manejo de los datos, ya que el formato "solo texto" no incluye, como su nombre indica, ningún tipo de formato. Una vez tenemos la muestra textual accesible y preparada para ser manipulada y poder trabajar con ella, procederíamos a la identificación de las unidades objeto de estudio en el trabajo de investigación específico.

\subsection{Incorporación de los resultados a una base de datos}

Una vez que cada unidad de estudio de la muestra textual ha sido clasificada y etiquetada, la podríamos incorporar a una base de datos creada utilizando la aplicación de Microsoft Excel, por considerar que la disposición de la información nos ayuda a una mejor organización de esta para una caracterización óptima de cada unidad y, además, nos ofrece mayor facilidad a la hora de operar estadísticamente con los datos.

Con el objetivo de ejemplificar cómo se podría llevar a cabo y su utilidad, proporcionamos algún ejemplo práctico tomando como unidad de análisis el mecanismo cohesivo de los marcadores discursivos. La búsqueda e identificación de los marcadores discursivos se realizaría de forma manual. En primer lugar, iríamos agregando a la base de datos las unidades de análisis identificadas en la muestra textual y seguidamente se procedería a su categorización atendiendo a ciertas variables que el investigador estime oportunas. En nuestro caso, los criterios de categorización de los marcadores discursivos han sido: el tipo al que pertenece según la clasificación seguida; la categoría gramatical; la posición inicial (I), media (M) o final (F) que ocupa el marcador respecto al segmento discursivo que introduce o al que acompaña; la relación, que hace referencia a la secuencia sintáctica o disposición de los segmentos discursivos a los que el marcador relaciona entre sí, invitando al receptor a establecer ese vínculo inferencial que caracteriza este tipo de unidades; su traducción (referida al equivalente utilizado en LM para su traducción, que no tiene necesariamente que coincidir con otro marcador discursivo); la categoría gramatical, posición, puntuación, relación, y tipo del marcador discursivo en la LM; así como el número de casos.

Con este tipo de información pensamos que se puede llegar a conocer con bastante precisión y profundidad el uso y la naturaleza cohesiva de los marcadores discursivos identificados en nuestra muestra textual, tanto en el original como en la traducción. En la base de datos, la información expuesta con anterioridad presenta este aspecto: 


\begin{tabular}{|c|c|c|c|c|c|c|c|c|c|c|c|c|}
\hline 1 & above all & Fraser: El & aborative $\mathrm{ma}$ & narker, Halli & iday \& Hasan: --; & Quirk et al: Listing & $g$-additive $-r$ & forcing & & & & \\
\hline 2 & Total: 1 & Extraido d & "Categoría & Posición & Puntuación & Relaciona & Traducción & Categoria gram Posición & Puntuación & Relaciona & Tipo (Martin \& & \& Núm casos \\
\hline 3 & Caso 1: & Arbitraje & sintagma a & tinicial & precedido por a & $S 1(0) ; M D, S 2(0)$ & sobre todo & sintagma prepo inicial & precedido por \& & S1 (o); MD, S2 (o) & No clasificado. & 1 \\
\hline $\begin{array}{l}4 \\
5\end{array}$ & & & & & & & & & & & & \\
\hline 6 & also & Fraser: El & aborative $\mathrm{ma}$ & רarker, Halli & iday \& Hasan: $\mathrm{AC}$ & dditive; Quirk et al. 1 & Listing - addit & - reinforcing & & & & \\
\hline 7 & Total: 1 & Extraído d & "Categoría & Posición & Puntuación & Relaciona & Traducción & Categoria gram Posición & Puntuación & Relaciona & Tipo (Martin \& & \& Núm casos \\
\hline 8 & Caso 1: & Arbitraje & adverbio $\mathrm{m}$ & & sin puntuación & $S 1(0) \cdot M D$ S2 (0) & asimismo & adverbio marca inicial & sin puntuación & S1 (0). MD S2 (0) & Estructurador o & \\
\hline$\frac{9}{10}$ & & & & & & & & & & & & \\
\hline 11 & alternatively & Fraser: $\mathrm{Cc}$ & ontrastive or & rElaborativ & ve marker, Hallid & lay \& Hasan: Additiv & ve - alternative & Ruirk et al.: Contrastive - repl & lace & & & \\
\hline 12 & Total 1: & Extraido d & "Categoria & Posición & Puntuación & Relaciona & Traducción & Categoria gram Posición & Puntuación & Relaciona & Tipo (Martin \& & \& Núm casos \\
\hline 13 & Caso 1: & Pagos inte & e adverbio $\mathrm{m}$ & rinicial & seguido de $\alpha, n$ & S1 (0) MD, S2 (0) & asimismo & adverbio marcar inicial & seguido de $\alpha, \downarrow$ & S1 (0). MD, S2 (o) & Estructurador: & ic \\
\hline 14 & & & & & & & & & & & & \\
\hline
\end{tabular}

Figura 6: Muestra de la base de datos I

\begin{tabular}{|c|c|c|c|c|c|c|c|c|c|c|c|}
\hline 44 & \multirow{3}{*}{\begin{tabular}{l|} 
at least \\
Total: 1 \\
Caso 1:
\end{tabular}} & \multicolumn{10}{|c|}{ raser:-; Halliday \& Hasan: Adversative; Quirk et al.: - } \\
\hline 45 & & \multirow{2}{*}{\multicolumn{2}{|c|}{$\begin{array}{l}\text { Extraído di Categoría Posición } \\
\text { Pagos inte sintagma r inicial }\end{array}$}} & \multirow{3}{*}{$\begin{array}{l}\text { Puntuación } \\
\text { precedido por p }\end{array}$} & \multirow{3}{*}{$\begin{array}{l}\text { Relación } \\
\text { p S1 (0) (MD S2 (0)) }\end{array}$} & \multirow{3}{*}{$\begin{array}{l}\text { Traducción } \\
\text { al menos }\end{array}$} & \multirow{2}{*}{$\begin{array}{l}\text { Categoría gram Posición } \\
\text { sintagma prepo inicial }\end{array}$} & \multirow{3}{*}{$\begin{array}{l}\text { Puntuación } \\
\text { precedido por }\end{array}$} & \multirow{3}{*}{$\begin{array}{l}\text { Relación } \\
\text { S1 (0) (MD S2 (0)) }\end{array}$} & \multicolumn{2}{|c|}{ Tipo (Martin \& Núm casos } \\
\hline 46 & & & & & & & & & & No clasificado. & 1 \\
\hline 47 & & & & & & & & & & & \\
\hline 49 & at this point & Fraser: -; Halliday \& $\mathrm{H}$ & Hasan: Ten & mporal; Quirk et & al: :- & & & & & & \\
\hline 50 & Total: 1 & Extraído diCategoria & Posición & Puntuación & Relación & Traducción & Categoría gram Posición & Puntuación & Relación & Tipo (Martin \& & Núm casos \\
\hline 51 & Caso 1: & Pagos inte sintagma r & & sin puntuación & S1 (o). S2 (0) MD. & a este respecto & sintagma prepo inicial & seguido de $\ll, »$ & $S 1(0) \cdot M D, S 2(0)$ & No clasificado. & 1 \\
\hline $\begin{array}{l}52 \\
53\end{array}$ & & & & & & & & & & & \\
\hline 54 & but & Fraser: Contrastive ma & larker; Hallic & iday \& Hasan: $\mathrm{AC}$ & iversative; Quirk et & tal.: - & & & & & \\
\hline 55 & Total: 1 & Extraido diCategoria & Posición & Puntuación & Relación & Traducción & Categoría gram Posición & Puntuación & Relación & Tipo (Martin \& & Núm casos \\
\hline 56 & Caso 1: & Pagos inte conjunción & ninicial & sin puntuación & S1 (0). MD S2 (0) & pero & conjunción marr inicial & seguida de $\alpha, »$ & $S 1(0) \cdot M D, S 2(0)$ & No clasificado. & 1 \\
\hline 57 & Caso 2: & Contratacic conjunción & nmedia & precedido por \& & \& $S 1(0) ; M D ~ S 2(0)$ & si bien & locución adverb media & entre comas & S1 (0), MD, S2 (0) & No clasificado. & 1 \\
\hline 58 & & & & & & & & & & & \\
\hline
\end{tabular}

Figura 7: Muestra de la base de datos II

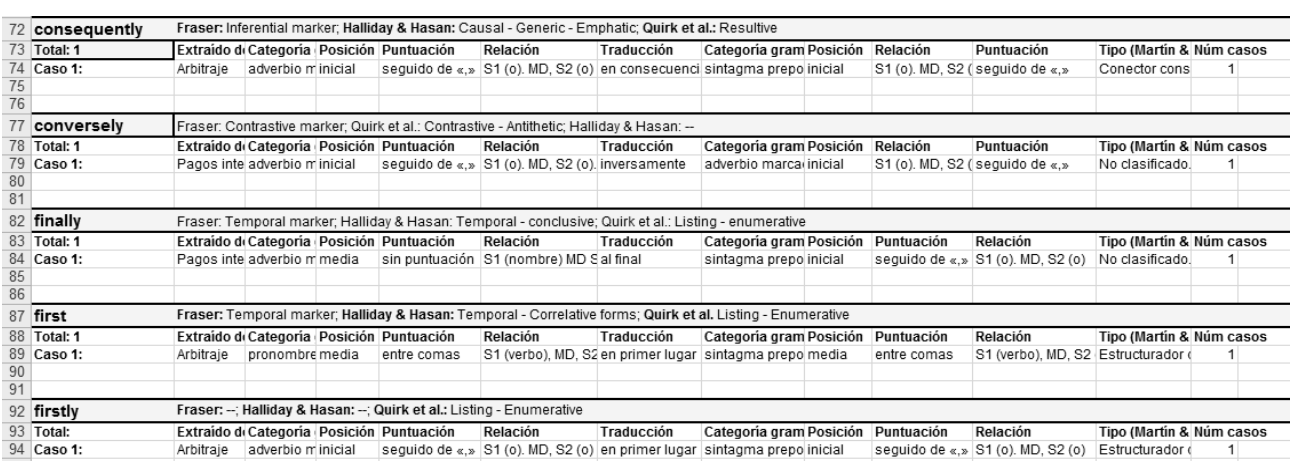

Figura 8: Muestra de la base de datos III

Una vez finalizada la base de datos que compila todas las unidades de estudio identificadas en la muestra textual original junto con su traducción y toda la información que les acompaña, procederíamos al análisis propiamente dicho de los datos que aportarían información muy valiosa relativa a las normas de traducción que operan en el proceso de trasvase, y, de esta manera, observar posibles tendencias recurrentes en su traducción, que, de haberlas, podrían aportar valiosos datos prácticos para los traductores del tipo de textos escogido en aras del establecimiento de una serie de rasgos contrastivos equiparables y/o distintivos que ayuden a los traductores en su labor.

Por poner un ejemplo concreto de los hallazgos encontrados tras el análisis e interpretación de los marcadores discursivos en una muestra textual formada por textos especializados de naturaleza jurídica emanados por organismos internacionales, destacamos la preferencia notable por la adecuación en la traducción, por lo que 
en el trasvase de este tipo de elementos parecen prevalecer los patrones retóricos (o convenciones textuales) del polo origen, lo cual se ha manifestado tanto en la tipología de los marcadores como en su posición, puntuación y segmentos discursivos que relacionan dichos marcadores en la muestra.

Además, la clara tendencia de la muestra textual hacia la adecuación como norma inicial de traducción hace que esta relativa abundancia de marcadores en los TO se reproduzca en los TM, lo cual difiere de los típicos rasgos discursivos de los textos jurídicos en español, en los que se encuentran relativamente pocos marcadores por ser ricos en un tipo de subordinación compleja caracterizada por la falta de una relación explícita entre las partes, lo que dificulta enormemente su lectura y comprensión. Por ello, se puede afirmar que el tipo de traducción "literal" que se ha aplicado ha redundado en un claro beneficio para los textos españoles, que muestran una organización e hilazón considerablemente mejores de lo que habrían tenido sin los marcadores "copiados" de sus originales en inglés.

\section{Conclusiones}

Este tipo de investigación se fundamentaría en la descripción de la práctica traductora en un contexto de especialidad, puesto que creemos que una teoría de la traducción ha de describir y explicar el funcionamiento real de la traducción a través de la observación y comparación rigurosa de originales y traducciones que refleje las decisiones que se toman durante el proceso de traducción.

El objetivo de este tipo de estudios es observar el trasvase interlingǘstico de ciertas unidades de estudio en el par de lenguas inglés-español en una muestra de textos conformada por originales y sus traducciones. El propósito es que los resultados de ese análisis sean de interés general en el ámbito de los estudios descriptivos de corte textual basados en el análisis de géneros profesionales y académicos y aplicados a un contexto especializado de traducción.

Como parte de la metodología empleada, aconsejamos introducir herramientas TAO en la investigación, en concreto en este caso, en el proceso de preparación de la muestra textual. Son herramientas que están al servicio del traductor, ayudándole en su labor de traducción. Igualmente, hemos podido comprobar con estudios previos, que también se pueden aplicar a una labor de investigación.

\section{Referencias bibliográficas}

AlcinA, A., «Translation Technologies: Scope, Tools and Resources», TARGET, International Journal of Translation Studies 20 (1) (2008), 79-102.

Amores Carredano, J.G., "Sistemas de Traducción Automática", Quark 19 (2000), 46-52. $<$ http://www.prbb.org/quark/19/019046.htm>

AustermüHL, F., Electronic tools for translators. Manchester: St. Jerome Publishing 2001.

Bermúdez BAusela, M., «Localización, tecnología y recursos informáticos aplicados a la traducción». Presentado en el Encuentro para traductores e intérpretes profesionales 
de Málaga. Organizado por: Educación digital y Nóvalo e-linguistic services 20/11/2010.

Even-Zohar, I., «Polysystem Theory». Poetics Today 1 (1-2) (1979), 287-310.

Hermans, T. (ed), The Manipulation of Literature: Studies in Literary Translation. Sydney: Croom \& Helm 1985.

Hutchins, J. And Somers, H. L., An Introduction to Machine Translation. London: Academic Press 1992. <http://www.hutchinsweb.me.uk/IntroMT-TOC.htm>

LAmbert, J. \& VAN GorP, H., «On Describing Translations», en Hermans, T., (ed.), The Manipulation of Literature: Studies in Literary Translation. Sydney: Croom \& Helm 1985, 42-53.

LEFEVERE, A., Translation, Rewriting and the Manipulation of Literary Fame. Londres: Routledge 1992.

LÓPEZ SÁNCHEZ, R., «Guía básica de software para traductores», Blog: Jugando a traducir. 2012. Disponible en: <http://jugandoatraducir.files.wordpress.com/2012/11/guiasoftware3.pdf $>$

Melby, A., «Eight Types of Translation Technology». Presentado en la American Translators Association ATA 39th Annual Conference. 4-9/11/1998. Hilton Head Island, South Carolina. $<$ http://www.ttt.org/technology/8types.pdf>

Neubert, A. \& Shreve, G. M., Translation as Text. Kent: Kent State University 1992.

Rabadán Álvarez, R., Equivalencia y traducción. Problemática de la equivalencia translémica inglés-español. León: Universidad de León 1991.

Santoyo Mediavilla, J.C. \& Rabadán Álvarez, R., «Basic Spanish Terminology for Translation Studies: A Proposal», Meta 36 (1) (1991), 318-322.

TourY, G., "The Nature and Role of Norms in Literary Translation» en Holmes, J.S.; Lambert, L. \& R. Van Den Broeck (eds.), Literature and Translation: New Perspectives in Literary Studies. Leuven: Arco 1978, 83-100.

Toury, G., Descriptive Translation Studies and Beyond. Amsterdam/Philadelphia: John Benjamins 1995.

Zetzsche, J., A Translator's Tool Box for the 21st Century. A Computer Primer for Translators. International Writers' Group 2003. 